\title{
Topological Phonon Modes in A Two-Dimensional Wigner Crystal
}

\author{
Wencheng $\mathrm{Ji}^{1}$ and Junren $\mathrm{Shi}^{1,2}$, 用 \\ ${ }^{1}$ International Center for Quantum Materials, Peking University, Beijing 100871, China \\ ${ }^{2}$ Collaborative Innovation Center of Quantum Matter, Beijing 100871, China
}

\begin{abstract}
We investigate the spin-orbit coupling effect in a two-dimensional Wigner crystal. We show that sufficiently strong spin-orbit coupling and an appropriate sign of $g$-factor could transform the Wigner crystal to a topological phonon system. We demonstrate the existence of chiral phonon edge modes in finite size samples, as well as the robustness of the modes in the topological phase. We explore the possibility of realizing the topological phonon system in two-dimensional Wigner crystals confined in semiconductor quantum wells/heterostructure. We find that the spin-orbit coupling is too weak for driving a topological phase transition in these systems. We argue that one may look for the topological phonon system in correlated Wigner crystals with emergent effective spin-orbit coupling.

PACS numbers: 63.20.-e, 73.21.-b
\end{abstract}

The concept of topology attracts great interest in recent investigations of condensed matter physics. The quantum Hall effect demonstrates a new state of matter which carries robust chiral edge modes protected by non-trivial topology of electron states. Recent research reveals various kind of topological insulators dictated by electron state topology in the presence of the time reversal symmetry or other symmetries [1]. Up to now, the studies are mainly focused on topological effects of the charge degree of freedom of electrons. On the other hand, the interest is recently extended to topological effects associated with collective excitations such as phonons and magnons [5-10]. Particularly, a topological phonon system (TPS) is predicted to possess robust chiral vibrational (phonon) modes at its edge [5]. These topological phonon modes (TPMs) are believed to be contributing to quantized thermal hall conductivity at low temperature [8. It is even postulated that TPMs are ubiquitous in biological systems and essential for a variety of processes in living organisms [5]. While these theoretical considerations are interesting and intriguing, we have yet to know an experimentally accessible and controllable way to construct a TPS in laboratory.

In this paper, we explore the possibility of realizing a TPS in a Wigner crystal (WC) of electrons. We investigate the effect of spin-orbit coupling (SOC) in a two-dimensional WC [11. We show that sufficiently strong SOC and an appropriate sign of $g$-factor could transform the WC to a TPS. We demonstrate the existence of chiral edge phonon modes in finite size samples, as well as the robustness of the chiral edge modes in the topological phase. We check the possibility in two-dimensional WCs confined in semiconductor quantum wells/heterostructure [12. We find that the SOC is too weak for driving a topological phase transition in these systems. We argue that one may look for the TPS in correlated WCs with emergent effective SOC.

It is well known that electrons form a WC in sufficiently low density when the Coulomb interaction dominates over the kinetic energy. Recent state-of-art Monte
Carlo calculations show that two-dimensional electrons undergo a series of phase transitions when lowering density, ultimately stabilized to a WC phase of a triangular lattice with ferromagnetic order of electron spins when $r_{s}>38$ [13 15], where $r_{s} \equiv 1 / \sqrt{\pi n_{e}} a_{B}$ is a dimensionless density parameter with electron density $n_{e}$ and effective Bohr radius $a_{B}$. Experimentally, two-dimensional WCs had been observed in ultra-clean 2D samples of GaAs/AlGaAs heterostructure [16].

We investigate phonon modes of a $2 \mathrm{D}$ WC of electrons. To model such a system, we consider a set of electrons with an effective mass $m^{*}$ arranged in a triangular lattice vibrating near their equilibrium positions. The Hamiltonian can be written as:

$$
H=\sum_{\boldsymbol{l}} \frac{\hat{\boldsymbol{P}}(\boldsymbol{l})^{2}}{2 m^{*}}+\Phi\left[\left\{u_{\alpha}(\boldsymbol{l})\right\}\right]+\sum_{\boldsymbol{l}} g^{*} \mu_{B} \hat{\sigma}_{\boldsymbol{l} z} B
$$

where the first term is the kinetic energy, the second term is the potential energy due to the Coulomb interaction between electrons, and the third term is the Zeeman energy due to a uniform magnetic field $B$ perpendicular to the $2 \mathrm{D}$ plane, with $\mu_{B}$ being Bohr magneton, $g^{*}$ being effective Land factor for the specific material hosting the $2 \mathrm{D}$ system, and $\hat{\sigma}_{l z}$ being the $z$-component of the Pauli spin matrices $\hat{\boldsymbol{\sigma}}_{\boldsymbol{l}}$ for an electron at site $\boldsymbol{l}$. For an electron subjected to both the magnetic field $\boldsymbol{B}$ and SOC, the kinetic momentum should be written as,

$$
\hat{\boldsymbol{P}}(\boldsymbol{l})=-i \hbar \nabla_{\boldsymbol{u}(\boldsymbol{l})}+\frac{1}{2} u(\boldsymbol{l}) \times e \boldsymbol{B}+\alpha_{0} \hat{\boldsymbol{\sigma}}_{\boldsymbol{l}} \times \boldsymbol{F}(\boldsymbol{l}),
$$

which involves two extra terms besides the canonical momentum operator, consisting of a vector potential term induced by the perpendicular magnetic field $\boldsymbol{B}$ and a SOC term induced by the electrostatic force $\boldsymbol{F}(\boldsymbol{l})$ acting on the electron by all other electrons in the system, respectively, and $u(\boldsymbol{l})$ is the displacement of an electron relative to its equilibrium position. The strength of the SOC is determined by a coefficient $\alpha_{0}$, which is a materialspecific parameter [17. Because all electrons are local- 
ized near their equilibrium positions in a $\mathrm{WC}$, it is sufficient to make a harmonic approximation to the potential energy:

$$
\Phi\left[\left\{u_{\alpha}(\boldsymbol{l})\right\}\right] \approx \Phi_{0}+\frac{1}{2} \sum_{\boldsymbol{l} l^{\prime}, \alpha, \beta} \Phi_{\alpha \beta}^{l-\boldsymbol{l}^{\prime}} u_{\alpha}(\boldsymbol{l}) u_{\beta}\left(\boldsymbol{l}^{\prime}\right),
$$

where $\alpha, \beta=x, y$, and the detailed form of the dynamic coefficients $\Phi_{\alpha \beta}^{l-l^{\prime}}$ for a triangular lattice is presented in Ref. [18. Correspondingly, the force $\boldsymbol{F}(\boldsymbol{l})$, which dictates the SOC, is related to the potential energy by:

$$
F_{\alpha}(\boldsymbol{l})=-\nabla_{u_{\alpha}(\boldsymbol{l})} \Phi=-\sum_{\boldsymbol{l}^{\prime}, \beta} \Phi_{\alpha \beta}^{\boldsymbol{l}, \boldsymbol{l}^{\prime}} u_{\beta}\left(\boldsymbol{l}^{\prime}\right) .
$$

To proceed, we need to make a further approximation. Equation (1) defines a system involving both phonons (vibration modes) and magnons (spin waves). However, since the WC has ferromagnetic order of spins and a perpendicular magnetic field will align and quench the spin degree of freedom, we can replace the Pauli spin matrices in the Hamiltonian with their mean field expectation values: $\left\langle\hat{\boldsymbol{\sigma}}_{\boldsymbol{l}}\right\rangle=(0,0, \sigma)$ with $\sigma=-\operatorname{sgn}\left(g^{*} B\right)$, because the direction of the electron spins will be parallel (anti-parallel) to the external magnetic field for $g^{*}<0\left(g^{*}>0\right)$.

We can then obtain equations of motion:

$$
\begin{aligned}
\dot{u}_{\alpha}(\boldsymbol{l}) & =\frac{\hat{P}_{\alpha}(\boldsymbol{l})}{m^{*}}, \\
\dot{\hat{P}}_{\alpha}(\boldsymbol{l}) & =-\sum_{\boldsymbol{l}^{\prime} \beta}\left[\Phi_{\alpha \beta}^{\boldsymbol{l - \boldsymbol { l } ^ { \prime }}} u_{\beta}\left(\boldsymbol{l}^{\prime}\right)+G_{\boldsymbol{l}-\boldsymbol{l}^{\prime}} \epsilon_{\alpha \beta} \hat{P}_{\beta}\left(\boldsymbol{l}^{\prime}\right)\right],
\end{aligned}
$$

where $\epsilon_{\alpha \beta}$ is antisymmetric tensor with $\epsilon_{x y}=1$, and

$$
G_{\boldsymbol{l}-\boldsymbol{l}^{\prime}}=\omega_{c} \delta_{\boldsymbol{l}, l^{\prime}}-\frac{\alpha_{0} \sigma}{m^{*}}\left[\Phi_{y y}^{\boldsymbol{l}-\boldsymbol{l}^{\prime}}+\Phi_{x x}^{l-\boldsymbol{l}^{\prime}}\right]
$$

where $\omega_{c}=e B / m^{*}$ is the magnetic cyclotron frequency of electrons.

A Fourier transformation recasts the equations of motion to an eigenvalue equation for phonon modes in a system breaking time-reversal symmetry [].

$$
\left[\begin{array}{cc}
0 & i \\
-i \Phi(\boldsymbol{k}) & G(\boldsymbol{k}) \sigma_{2}
\end{array}\right] \psi_{n}(\boldsymbol{k})=\omega_{n}(\boldsymbol{k}) \psi_{n}(\boldsymbol{k}),
$$

where $\boldsymbol{k}$ is defined in the Brillouin zone for the triangular lattice, $\psi_{n}(\mathbf{k})=(\boldsymbol{u}(\boldsymbol{k}), \boldsymbol{P}(\boldsymbol{k}))^{T}, \sigma_{2}$ is the second Pauli matrix, and $\Phi(\boldsymbol{k}), G(\boldsymbol{k}), \boldsymbol{u}(\boldsymbol{k})$, and $\boldsymbol{P}(\boldsymbol{k})$ are Fourier transformations of $\Phi\left(\boldsymbol{l}-\boldsymbol{l}^{\prime}\right) / m^{*}, G_{\boldsymbol{l}-\boldsymbol{l}^{\prime}}, m^{*} \boldsymbol{u}(\boldsymbol{l})$, and $\boldsymbol{P}(\boldsymbol{l})$, respectively. We have:

$$
G(\boldsymbol{k})=\omega_{c}-\alpha_{0}\left(\Phi_{x x}(\boldsymbol{k})+\Phi_{y y}(\boldsymbol{k})\right) \sigma .
$$

We note that Eq. (8) will give rise to four modes for each $\boldsymbol{k}$ with two positive and two negative frequency branches. However, the negative frequency branches can be related to the positive frequency branches by $\omega_{n}^{-}(\boldsymbol{k})=-\omega_{n}^{+}(-\boldsymbol{k})[\underline{8}$. In the following, we will only show the positive frequency branches.

Figure 11 shows the evolution of phonon dispersion along high symmetry lines of the Brillouin zone for different strengths of SOC in the presence of a magnetic field. We observe that the uniform magnetic field induces gaps between the two branches of phonon modes at both $\Gamma$ and $\boldsymbol{K}\left(\boldsymbol{K}^{\prime}\right)$ points of the Brillouin zone, as shown in Fig. 1(a). In increasing the strength of SOC, the gap at $\boldsymbol{K}\left(\boldsymbol{K}^{\prime}\right)$ point is gradually closed and reopened, while the gap at $\Gamma$-point is unaffected, as shown in Fig. 1 (b)-(d). Such behavior is similar to what happens in a topological insulator, in which a closing and a reopening of a gap indicates a band inversion which transforms a normal insulator to a topological insulator. We thus expect that the evolution observed here may also drive a topological phase transition, albeit for the phonon bands.
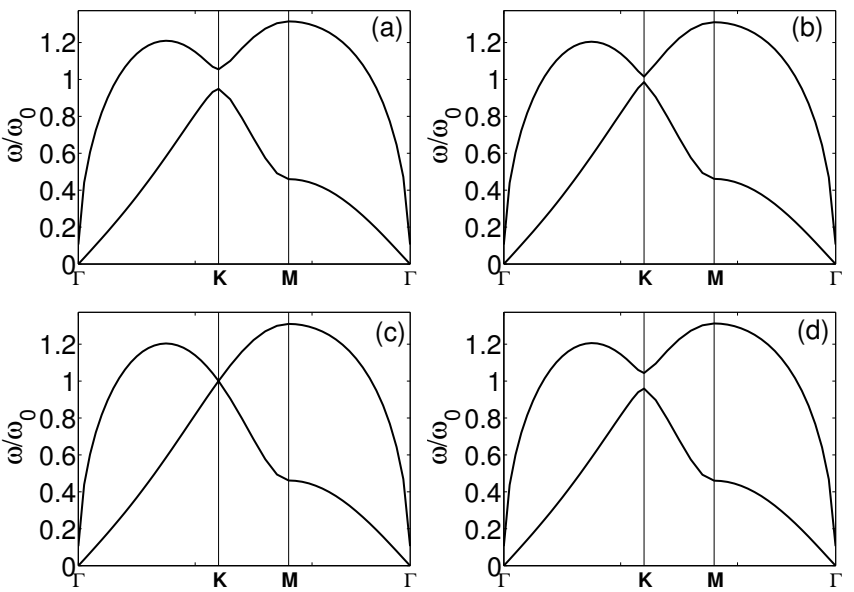

FIG. 1. Phonon dispersion along high symmetry lines of the Brillouin zone for different strengths of SOC. We set $\omega_{c}=$ $0.1 \omega_{0}$. The strengths of SOC are: (a) $\alpha_{0}=0$; (b) $\alpha_{0} \omega_{0}=0.04$; (c) $\alpha_{0} \omega_{0}=0.05 ;$ (d) $\alpha_{0} \omega_{0}=0.1$. The effective Land factor is assumed to be negative: $g<0$.

To confirm the topological change of the phonon bands, we calculate the phonon Berry curvature that is defined as:

$$
\boldsymbol{\Omega}_{n}(\boldsymbol{k})=-\operatorname{Im}\left[\frac{\partial \bar{\psi}_{n}(\boldsymbol{k})}{\partial \boldsymbol{k}} \times \frac{\partial \psi_{n}(\boldsymbol{k})}{\partial \boldsymbol{k}}\right]
$$

where $\bar{\psi}_{n}(\boldsymbol{k}) \equiv \psi_{n}^{\dagger}(\boldsymbol{k})\left[\begin{array}{cc}\Phi(\boldsymbol{k}) & 0 \\ 0 & I_{2 \times 2}\end{array}\right][$ [ $]$. Figure 2 shows the distribution of the Berry curvatures for the upper phonon bands before and after the band inversion. It can be clearly seen that the Berry curvature peaks at $\Gamma$ and $\boldsymbol{K}\left(\boldsymbol{K}^{\prime}\right)$. For weak SOC, the Berry curvatures at $\Gamma$ and $\boldsymbol{K}\left(\boldsymbol{K}^{\prime}\right)$ have opposite signs, resulting in cancellation of the Chern number that is proportional to an integration of the Berry curvature over the whole Brillouin zone. On the other hand, the Berry curvatures at $\Gamma$ and $\boldsymbol{K}\left(\boldsymbol{K}^{\prime}\right)$ have the same signs after the band inversion, indicating 

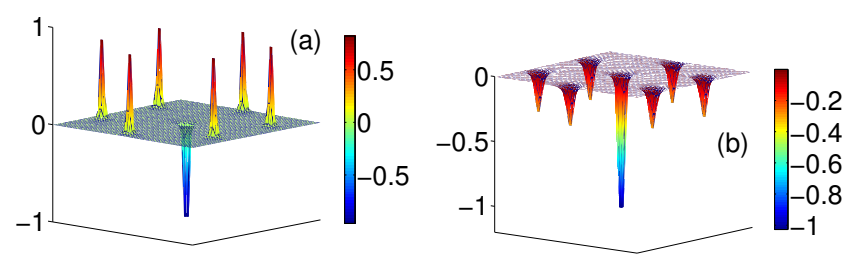

FIG. 2. (color online) Distribution of the Berry curvatures for the upper phonon bands before and after the band inversion. The parameters are the same as those in Fig. 1(b) and Fig. 1.(d), respectively.

the topological change of the phonon band. We calculate the Chern numbers for both cases, and obtain $C=0$ and $C=-2$ for bands before and after the inversion, respectively.

The behavior can be easily understood. To see that, we recast the eigenvalue equation (8) to an equivalent $2 \times 2$ hermitian eigenvalue problem:

$$
\hat{h}(\boldsymbol{k}) \mathbf{u}(\boldsymbol{k}) \equiv\left(\Phi(\boldsymbol{k})+\omega G(\boldsymbol{k}) \sigma_{2}\right) \mathbf{u}(\boldsymbol{k})=\omega^{2} \mathbf{u}(\boldsymbol{k}) .
$$

Near the $\boldsymbol{K}$ point, we can expand $\hat{h}(\boldsymbol{k}) \approx h_{0}+\lambda_{1} \triangle k_{y} \sigma_{1}-$ $\lambda_{2} \triangle k_{x} \sigma_{3}+M \sigma_{2}$, where $h_{0}, \lambda_{1}, \lambda_{2}$ are constants, and

$$
M=\omega G(\boldsymbol{K})=\omega\left(\omega_{c}-2 \alpha_{0} \sigma \omega_{0}^{2}\right),
$$

where $\omega_{0}=\sqrt{\Phi_{x x}(\boldsymbol{K})}$ is the phonon frequency at $\boldsymbol{K}$ point in the absence of both the magnetic field and SOC, and is related to material parameters by $\hbar \omega_{0}=$ $1.967 r_{s}^{-3 / 2} \mathrm{Ry}^{*}$, where $\mathrm{Ry}^{*}$ is the effective Rydberg of the hosting semiconductor [17]. It is easy to see that the effective hamiltonian has the same form as the 2D massive Dirac hamiltonian with the mass $M$. The inversion of the bands and the topological phase transition occur when $M$ changes sign. This is possible only when $g^{*} \alpha_{0}<0$. In this case, $M$ changes sign when

$$
2 \alpha_{0} \omega_{0}=-\operatorname{sgn}\left(g^{*}\right) \frac{\omega_{c}}{\omega_{0}} .
$$

On the other hand, the Berry curvature near the $\Gamma$ point is only determined by the external magnetic field and is not affected by the SOC, because $\Phi_{\alpha \beta}(\boldsymbol{\Gamma})=0$ and $G(\boldsymbol{\Gamma})=\omega_{c}$. Figure 3 shows the corresponding phase diagram.

For a TPS, there exist TPMs in a finite-size sample. To show that, we consider a strip of WC along the $x$ direction and calculate phonon dispersion as a function of $k_{x}$. Figure 4 shows the edge states for a few representative cases. We find that there exist edge states for both the topologically trivial phase (a, c) and the topologically non-trivial phase (b, d). The difference between the two phases is obscured when the magnetic field is not strong enough for opening a full gap in the phonon spectrum $(\mathrm{a}, \mathrm{b})$. In this case, we can find two counter-propagating

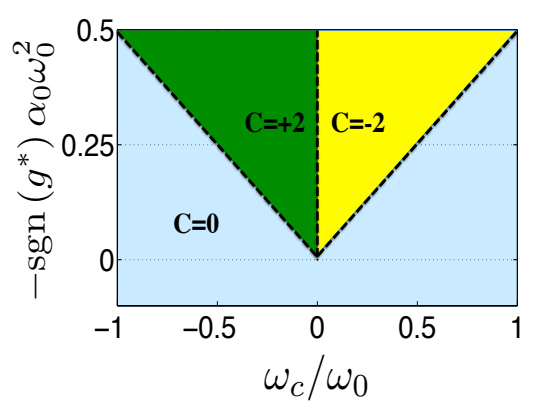

FIG. 3. (color online) Phase diagram for the phonon bands of a two-dimensional ferromagnetic WC. The regions with nonzero Chern-number is topologically nontrivial.

modes at each of the edges for both the phases. However, only one of the modes survives in the gap regime near $\boldsymbol{K}\left(\boldsymbol{K}^{\prime}\right)$ point. The topological phase transition is accompanied by a change of the propagating direction of the surviving edge mode.

The topological difference becomes apparent when we increase the strength of the magnetic field to open a full gap in the phonon spectrum (c, d). In this case, the edge modes completely disappear in the gap regime for the topologically trivial phase, while for the topologically non-trivial phase, there are two chiral edge modes propagating along the same direction. The two chiral edge modes are consistent to the Chern number $C=-2$. One can also clearly see that both the edge modes emerge from $\boldsymbol{\Gamma}$ point, and end near $\boldsymbol{K}\left(\boldsymbol{K}^{\prime}\right)$ point. The topological difference between the two phases lies in the different ways that the edge modes connect the bulk phonon bands. For the topological trivial phase, the edges modes connect the same phonon band, while for the topological non-trivial phase, the edge modes make inter-band connections.

We explore the possibility of realizing the TPS in WCs of real materials. We first check WCs formed in two-dimensional electron gases confined in semiconductor quantum wells/heterostructure. Some of the hosting semiconductors, such as $\mathrm{AlSb}, \mathrm{Al}_{x} \mathrm{In}_{1-x} \mathrm{As}, \mathrm{InP}$, and ZnSe, can be ruled out because their parameters $\alpha_{0}$ and $g$ have the same sign. On the other hand, other semiconductors such as GaAs, AlAs, InAs, InSb do have opposite signs for $\alpha_{0}$ and $g$. Table $\mathbb{\Pi}$ shows relevant parameters for them. Unfortunately, we find that the SOC is too weak for all of these materials. For a WC stabilized purely by the electron-electron interaction, SOC induced gaps $\Delta_{K}$ are of the order of $10^{-7} \mathrm{meV}$, and the magnetic field must be weaker than $10^{-7} \mathrm{~T}$ for a TPS. This is apparently impossible for real world experimental conditions.

We also explore the possibility in hole systems. In this case, the SOC has a different form due to the band symmetry [17. As a result, the coupling between the momentum and spin is proportional to $\left[p_{x}(\boldsymbol{l}) E_{y}(\boldsymbol{l})+p_{y}(\boldsymbol{l}) E_{x}(\boldsymbol{l})\right] \sigma$, instead of $\left[p_{x}(\boldsymbol{l}) E_{y}(\boldsymbol{l})-\right.$ $\left.p_{y}(\boldsymbol{l}) E_{x}(\boldsymbol{l})\right] \sigma$ for an electron system. It gives rise to a dif- 

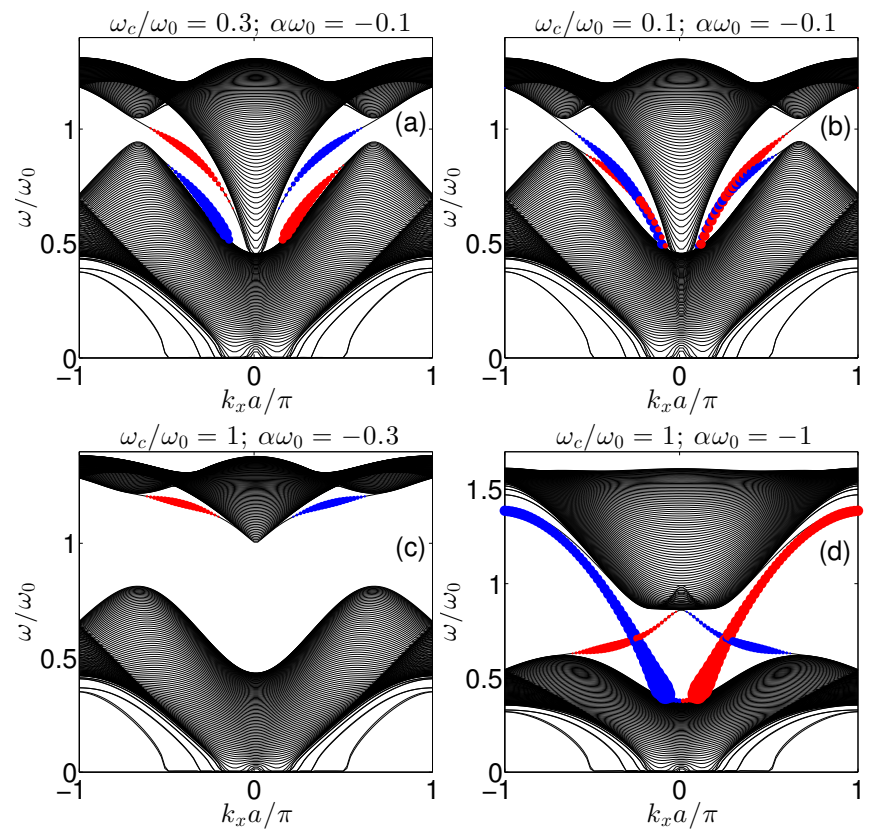

FIG. 4. (color online) Phonon dispersion of a finite size sample with different values of $\alpha \omega_{0}$ and $\omega_{c} / \omega_{0}$. The finite size sample is extended along the $x$ direction, and has a width of 100 sites in the $y$-direction. The red (blue) dots represent modes localized at the top (bottom) edge of the sample. The size of a dot indicates the degree of localization that is proportional to a quantity $e_{n}=\sum_{i}\left|\psi_{n}(i)\right|^{4}$, a larger value of which corresponds to a more localized edge mode.

\begin{tabular}{|l||c|c|c|c|c|c|}
\hline & $g^{*}$ & $\begin{array}{c}\mathrm{Ry} \\
(\mathrm{meV})\end{array}$ & $\begin{array}{c}\alpha_{0} / \hbar \\
\left(\mathrm{eV}^{-1}\right)\end{array}$ & $\begin{array}{c}\hbar \omega_{0}^{r_{s}}=38 \\
(\mathrm{meV})\end{array}$ & $\begin{array}{c}\Delta_{K}^{r_{s}=38} \\
(\mathrm{neV})\end{array}$ & $\begin{array}{c}B_{c}^{r_{s}=38} \\
(\mu \mathrm{T})\end{array}$ \\
\hline \hline $\mathrm{GaAs}$ & - & 5.882 & 0.0455 & 0.0494 & 0.221 & 0.127 \\
\hline $\mathrm{AlAs}$ & + & 20.157 & -0.00479 & 0.169 & 0.274 & 0.356 \\
\hline $\mathrm{InAs}$ & - & 1.461 & 0.352 & 0.0123 & 0.106 & 0.0210 \\
\hline $\mathrm{InSb}$ & - & 0.590 & 0.955 & 0.00495 & 0.0469 & 0.00563 \\
\hline
\end{tabular}

TABLE I. Parameters calculated for a number of semiconductors. Material parameters are adopted from Ref. 17, with $\alpha_{0} \equiv\left(m^{*} / \hbar e\right) r_{41}^{6 c 6 c}$. Ry* is the effective Rydberg for the material, $\Delta_{K} \equiv 2 \hbar \alpha_{0} \omega_{0}^{2}$ is the gap induced by SOC at $\boldsymbol{K}$ point, and $B_{c}$ is the critical strength of the magnetic field for the topological phase transition. The values of $\omega_{0}, \Delta_{K}$ and $B_{c}$ at $r_{s}=38$ are shown, as indicated by the superscripts. The values of these quantities at other density can be determined by: $\omega_{0}=\omega_{0}^{r_{s}=38}\left(38 / r_{s}\right)^{3 / 2}, \Delta_{K}=\Delta_{K}^{r_{s}=38}\left(38 / r_{s}\right)^{3}$, $B_{c}=B_{c}^{r_{s}=38}\left(38 / r_{s}\right)^{3}$.

ferent $G(\boldsymbol{k})=e B / m+\alpha_{0}\left(D_{y y}(\mathbf{k})-D_{x x}(\mathbf{k})\right) \sigma$, in which the SOC contribution vanishes at $\boldsymbol{K}$ point. Therefore, the SOC in a hole system cannot drive a topological phase transition of phonon.

We also explore the possibility in WCs stabilized by a strong magnetic field, which quenches the kinetic energy of electrons and favors the formation of WCs [19, 20]. These WCs could be stabilized in quantum Hall systems at much higher electron densities 21. For a typical elec- tron density with $r_{s} \sim 1$, we find that (see Table $\mathbb{I}$ ) for GaAs, $\Delta_{K} \sim 12 \mu \mathrm{eV}$ and $B_{c} \sim 7 \mathrm{mT}$. Other semiconductors have parameters in similar orders of magnitude. They are still too small to provide observable physical effects.

All summarized, we conclude that WCs in real semiconductor materials cannot support a TPS. This is not surprising because as a relativistic effect, the SOC is always weak. From Table I, we see that the strength of the SOC must be enhanced at least four orders of magnitude to reach $\alpha_{0} \omega_{0} \sim 1$, a magnitude necessary for a clear manifestation of the topological effect. This is unfortunately impossible in real world.

We argue that one may look for the TPS in WCs with emergent effective SOC. Actually, SOC is only one of many possible forms of coupling between orbital motion and internal degrees of freedom. The strengths of other forms of the coupling are not necessarily constrained by the relativistic principle, and could be potentially very strong. An interesting and potentially relevant case could be found in the fractional quantum Hall systems, in which a new species of WCs, i.e., Wigner crystals of composite fermions, may form 22. In these systems, the orbital motion of electrons is strongly entangled with degrees of freedom of all other electrons in the system due to the strong-correlation nature of the state. One would expect that the entanglement serves as effective SOC, and gives rise to similar effects as those predicted in this paper. The effective SOC emerges from the strong correlation, and its strength is not constrained by the relativistic principle. This possibility will be left for further investigations.

This work is supported by National Basic Research Program of China (973 Program) Grant No. 2015CB921101 and National Science Foundation of China Grant No. 11325416.

* junrenshi@pku.edu.cn

[1] C. L. Kane and E. J. Mele, Phys. Rev. Lett. 95, 226801 (2005).

[2] C. L. Kane and E. J. Mele, Phys. Rev. Lett. 95, 146802 (2005).

[3] B. A. Bernevig, T. L. Hughes, and S. C. Zhang, Science 314, 1757 (2006).

[4] M. Knig, S. Wiedmann, C. Brne, A. Roth, H. Buhmann, L. Molenkamp, X.-L. Qi, and S.-C. Zhang, Science 318, 766 (2007).

[5] E. Prodan and C. Prodan, Phys. Rev. Lett. 103, 248101 (2009).

[6] L. Zhang, J. Ren, J.-S. Wang, and B. Li, Phys. Rev. Lett. 105, 225901 (2010).

[7] N. Berg, K. Joel, M. Koolyk, E. Prodan, Phys. Rev. E 83, 021913 (2011).

[8] T. Qin, J. Zhou, and J. Shi, Phys. Rev. B 86, 104305 (2012). 
[9] C. Strohm, G. L. J. A. Rikken, and P. Wyder, Phys. Rev. Lett. 95, 155901 (2005).

[10] A. V. Inyushkin and A. N. Taldenkov, JETP Lett. 86, 379 (2007).

[11] Yu. P. Monarkha and V. E. Syvokon, Low Temperature Physics 38, 1067 (2012).

[12] T. Ando, A. B. Fowler, and F. Stern, Rev. Mod. Phys. 54, 437 (1982).

[13] B. Tanatar and D. M. Ceperley, Phys. Rev. B 39, 5005 (1989).

[14] C. Attaccalite, S. Moroni, P. Gori-Giorgi, and G. B. Bachelet, Phys. Rev. Lett. 88, 256601 (2002).

[15] N. D. Drummond and R. J. Needs, Phys. Rev. Lett. 102, 126402 (2009).

[16] J. Yoon, C. Li, D. Shahar, D. C. Tsui, M. Shayegan, Phys
Rev Lett 82, 1744 (1999).

[17] R. Winkler, Spin-Orbit Coupling Effects in TwoDimensional Electron and Hole Systems (Springer, 2010).

[18] L. Bonsall and A. A. Maradudin, Phys. Rev. B 15, 1959 (1979).

[19] H. Fukuyama, P. M. Platzman, and P. W. Anderson, Phys. Rev. B 19, 5211 (1979).

[20] K. Maki and X. Zotos, Phys. Rev. B 28, 4349 (1983).

[21] H. Zhu, Y. P. Chen, P. Jiang, L. W. Engel, D. C. Tsui, L. N. Pfeiffer, and K. W. West, Phys. Rev. Lett. 105, 126803 (2010).

[22] A. C. Archer, K. Park, and J. K. Jain, Phys. Rev. Lett. 111, 146804 (2013). 